\title{
Factors influencing nurses' knowledge and attitudes toward patients in chronic pain with opioid use disorder: A literature review
}

\author{
Suha Abdulwahab*1, Vahe Kehyayan ${ }^{1}$, Atef Al-Tawafsheh ${ }^{2}$ \\ ${ }^{1}$ University of Calgary in Qatar, Doha, Qatar \\ ${ }^{2}$ Hamad Medical Corporation, Qatar
}

Received: April 8, 2020

DOI: $10.5430 /$ jnep.v10n9p37
Accepted: May 22, 2020

Online Published: May 27, 2020

\begin{abstract}
Background and objective: Chronic pain is a common symptom among patients worldwide. This issue leads health care professionals to manage patients' chronic pain by using opioids. However, some patients tend to abuse these medications and develop opioid use disorder. The aim of the study was to identify and explore factors that influence nurses' knowledge and attitudes toward patients in chronic pain with opioid use disorder.

Methods: A literature review was conducted. CINAHL, Medline, and PsychINFO databases were used to search for relevant articles. A total of 12 articles that met the inclusion criteria were retrieved.

Results: This literature review showed several factors that influence nurses' knowledge and attitudes. These factors were nurses' education, role support, demographic factors, nurses' experiences, and nurses' beliefs and culture.

Conclusions: The findings of this literature review will inform the development of an educational program to promote nurses' knowledge and attitudes toward patients in chronic pain with opioid use disorder.
\end{abstract}

Key Words: Substance abuse, Opioid abuse, Opioid use disorder, Chronic pain, Pain management, Nursing attitudes, Knowledge

\section{INTRODUCTION}

Chronic pain is the most common reason to seek health care services. Approximately $30 \%$ to $40 \%$ of people worldwide suffer from chronic pain. ${ }^{[1]}$ The prevalence of chronic pain in the Gulf region, particularly in Kuwait is high and it was estimated to be $55.3 \%$ between 2014 and 2015. ${ }^{[2]}$ Health care providers prescribe opioids to control chronic pain in their patients. Hagemeier (2018) ${ }^{[3]}$ stated that one in five patients with chronic pain is treated with opioids in the United States. This author reported also that in 2016, approximately $66.5 \%$ opioid prescriptions were prescribed for 100 Americans. However, the World Health Organization reported that
27 million patients misused opioids in $2016 .{ }^{[4]}$ Opioids use in chronic pain management often increases the risk of opioid use disorder (OUD). In the Middle East, the rate of opioid abuse has been increasing and OUD has been reported to be prevalent in the Gulf Region. A study by Alam-mehrjerdi, Noori, and Dolan (2016) ${ }^{[5]}$ reported that in Oman 1,945 men and women used illicit drugs including opioids between 2006 and 2011.

The increase of opioid abuse burdens the health care system and presents a challenge to health care professionals, especially nurses, about identifying and managing OUD.

*Correspondence: Suha Abdulwahab; Email: SAbdo1@ @amad.qa; Address: University of Calgary in Qatar, Doha, Qatar. 
Most nurses who encounter patients with OUD lack knowledge about and have negative attitudes toward them, such as stigma, bias, discrimination, and neglect. ${ }^{[6]}$ These behaviors and attitudes lead to the major negative consequence of undertreating these patients' pain or providing poor quality of care to them. These behaviors also may prevent opioid users from seeking the needed health care, which could result in more complications in their conditions. ${ }^{[6]}$ The purpose of this paper was to conduct a literature review to identify and explore factors that influenced nurses' knowledge and attitudes toward patients in chronic pain with OUD and to provide insight into the gap in nurses' current knowledge of OUD and chronic pain.

\subsection{Background}

Substance use disorder (SUD) is an umbrella term for different types of addiction to certain substances, such as alcohol, illicit drugs, and opioids. Other terms are interchangeably used with SUD in the literature, such as substance abuse, drug abuse, drug dependence, and OUD. ${ }^{[7,8]}$ The use of these terms is influenced by different contexts, such as culture, politics, and geographic factors. ${ }^{[7]}$ Because the focus of this paper is on prescribed opioids rather than other drugs, the term OUD was used. Mascola, Ann, Borders, Mishka, and Terplan (2017) ${ }^{[9]}$ defined OUD as the continuous use of opioid drugs harmfully despite knowing their adverse outcomes. Pain can be categorized into acute and chronic pain. As most opioid abusers suffer from chronic pain, ${ }^{[3]}$ the focus of this paper is on chronic pain. Chronic pain is defined as "pain lasting for 3 months or beyond the time of normal tissue healing" (p. 201). ${ }^{[3]}$ Some patients with chronic pain misuse their prescribed opioids by combining different types of drugs or self-adjusting of doses without physicians' knowledge. ${ }^{[10]}$ Some patients abuse prescribed opioids for reasons other than medical purposes, such as self-pleasure or euphoric feelings. ${ }^{[11]}$

\subsection{Patients' behaviors related to opioid therapy}

Several behaviors were highlighted in the literature to identify patients in chronic pain with OUD. These behaviors include multiple late or missed appointments, forging prescriptions, reporting lost prescriptions, resisting changes in medication therapy, and reporting allergies or ineffective pain relief to any non-opioid analgesics, stealing or borrowing medication from others and seeking medications from different health care professionals. ${ }^{[12]}$ Ashley $(2008)^{[13]}$ also reported on patients' behaviors of opioid abuse including running out of medication before follow-up appointments, self- adjusting medication dosing, aggressively demanding more drugs, and suggesting medication options to the health care provider. Other behaviors indicative of opioid abuse include refusal of physical examination, doing diagnostic tests, and providing medical history, or providing unclear medical histories. ${ }^{[14]}$ Some patients on opioid therapy for their chronic pain may develop similar behaviors of OUD. Therefore, nurses should be familiar with symptoms that are commonly associated with patients on opioid therapy especially tolerance, withdrawal, physical dependence, pseudoaddiction, and opioid induced hyperalgesia.

\subsection{Monitoring patients on opioid therapy}

Strategies are required to monitor patients' behaviors related to opioid therapy to eliminate the risk for opioid abuse. According to Cheatle et al. (2014) ${ }^{[10]}$ patients' behaviors related to addiction may be indicative of their need for adequate management of their chronic pain or drug abuse. Therefore, health care providers should interview such patients without judgmental manner. Any change in the patient's therapy or discontinuations of the opioid should depend on the interview result. The Substance Abuse and Mental Health Services Administration in the United States has developed a guideline that emphasizes all opioid treatment interventions should provide adequate testing and analyzing any signs of opioid abuse ${ }^{[10]}$ Health care providers must take a comprehensive history and conduct a physical assessment of patients who started opioid therapy. ${ }^{[15]}$ At the patient's initial visit to the doctor, a standardized written agreement that outlines the mutual responsibilities of the health care provider and patients should be used. Based on the initial assessment, patients who are at low risk of opioid abuse should be monitored periodically during each visit and follow up every six months, while patients at medium risk should be monitored during each visit and follow up every three months. ${ }^{[15]}$ Hagemeier $(2018)^{[3]}$ stated that patients with suspected OUD should be diagnosed by using the criteria of Diagnostic and Statistical Manual of Mental Disorders, fifth edition (DSM-5). This criterion includes 11 items and patients with OUD must have at least two items over 12 months of assessment.

\subsection{Management of chronic pain in patients with OUD}

Different interventional methods are available to prevent opioid abuse and its adverse consequences. Effective chronic pain management is the initial step for opioid abuse prevention. ${ }^{[11]}$ This management may include pharmacotherapy, non-pharmacotherapy, and interventional management. Pharmacotherapy of chronic pain consists of opioids such as morphine, and non-opioid analgesics such as antidepressant and antiepileptic drugs depending on the etiology of the pain. ${ }^{[3,10]}$ Some patients with chronic pain and active OUD need to receive treatment for their addiction before pain management begins because the regular abuse of psychoactive drugs results in physiological disruption, which may affect

ISSN 1925-4040 E-ISSN 1925-4059 
the effectiveness of pain treatment. ${ }^{[10]}$ Other methods of treating patients with chronic pain and opioid abuse is tapering their medications gradually and referring them to opioid treatment program or addiction specialist. ${ }^{[10]}$ Interventional pain management is another method for treating chronic pain that includes epidural steroid injections, radiofrequency neurotomy, spinal cord stimulation, and sacroiliac joint injections. ${ }^{[10]}$ The use of complementary and alternative medicine is also an appropriate option for pain management. ${ }^{[10]}$ These options include acupuncture, exercise, physical therapy, and cognitive therapy. ${ }^{[10,11,16]}$

\subsection{Nurses' role in the management of patients in chronic pain with OUD}

Working with patients in chronic pain who are on opioid therapy is a challenge in nursing practice. Nurses have a crucial role to develop a comprehensive approach to care for these patients. Griffiths $(2017)^{[17]}$ suggested that an element of this comprehensive approach is providing high quality and safe care to patients in chronic pain with opioid abuse. Nurses also should be knowledgeable about the complexity of chronic pain and opioid abuse because this affects patients' neurological, psychosocial, and spiritual aspects and as well develops maladaptive behaviors. ${ }^{[17]}$ Patients in chronic pain with opioid abuse present with different symptoms, which can be difficult for nurses to recognize. Hudspeth $(2016)^{[18]}$ recommended that it is essential for nurses to use screening tools to evaluate patients' intensity of pain and recognize those with opioid abuse. The use of these screening tools also helps nurses to identify patients who are at risk of opioids abuse. Health history should be taken as well including any current or past use of opioids, history of mental illness, and patients' and their families' histories of substance abuse. Nurses should involve psychiatrists and pain management teams if patients have any psychiatric disorders or are being treated for chronic pain. ${ }^{[17]}$ After physical assessment and medical history, nurses play an important role in practicing evidence-based pain management and using alternative medicines rather than opioids in treating pain based on individual's needs. ${ }^{[10]}$ Another important role for nurses is to educate patients about opioid use, its side effects, and safe disposal of medications. ${ }^{[17]}$

However, nurses' role may be negatively affected by their lack of knowledge and negative attitudes toward patients with opioid abuse in chronic pain. Some nurses may have discriminatory attitudes by labeling some patients in chronic pain as opioid abusers. Consequently, these patients may receive inadequate health care and pain management than those who are not labeled as abusers. ${ }^{[7]}$

Published by Sciedu Press

\subsection{Impact of opioid use disorder}

Opioid use disorder has serious effects on individuals' lives, their families as well as health care systems. Hagemeier $(2018)^{[3]}$ stated that patients' quality of life could be compromised because opioids abuse affects patients' physical illness and mental health. The risk of developing physical illness in this population is higher than other populations. These physical illnesses include infections and transmissible diseases, such as cellulitis, endocarditis, and hepatitis. ${ }^{[3]}$ In severe cases, coma or death may also result from opioid overdoses. ${ }^{[18]}$ Opioid use disorder also affects the individual's mental health by causing sleep disturbances, mood swings, irritability, anxiety, and depression. ${ }^{[3]}$ Individuals with opioid abuse become increasingly isolated from their family and society. The productivity of their work may be even diminished because of frequent absenteeism, which may lead to the termination of employment. ${ }^{[19]}$ Opioid abuse in individuals also affects their families in several domains, such as financial, lifestyle, personal interactions, and relationships as well as physical and mental health of the family. ${ }^{[3]}$

Opioid use disorder is a global issue that burdens health care systems in different dimensions. For example, it increases the cost of health care services for these individuals, such as providing necessary diagnostic procedures and repeated hospital admissions. ${ }^{[18]}$ In the United States, the mean cost of health care for patients with opioid abuse is US \$14,054 to US $\$ 20,546$ annually. ${ }^{[3]}$ These patients also frequently use other health care resources, such as occupying hospital beds, increasing the waiting times for other patients, and overloading outpatient clinics.

\section{METHOD}

A literature review was conducted for the purposes of the study. A literature review is defined as an "objective, thorough summary and critical analysis of the relevant available research and non-research literature on the topic being studied" (Cronin, Ryan, and Coughlan (2008) p. 38). ${ }^{[20]}$ The keywords that were used to search databases included: substance abuse*, opioid abuse*, opioid use disorder* drug use disorder*, chronic pain, pain management, nurs*, attitude*, and knowledge. The following databases were searched: CINAHL, MEDLINE, and PsychINFO. The initial search resulted in 1,217 articles. The following limiters were used: articles published between 2008 and 2019, English language, and peer review. After applying the limiters 588 articles were identified for possible inclusion. The titles and abstracts of the retrieved articles were screened. The screening resulted in excluding 509 articles. The remaining 79 articles were subjected to full text review. Duplicate articles were removed $(n=10)$. The full text review of the remaining 69 articles 
eliminated a further 57 articles based on the inclusion and exclusion. The most common reason for exclusion was articles published about OUD in patients with acute pain or chronic cancer pain. A total of 12 articles were determined to be suitable for inclusion in this literature review.

The 12 articles were critically appraised using the Mixed Method Appraisal Tool (MMAT), version 2018. The MMAT is a critical appraisal tool aimed to review systematic mixed studies that include qualitative, quantitative and mixed methods studies. ${ }^{[21]}$ The MMAT consists of two parts: a checklist of criteria and an explanation of these criteria. The checklist specifies the category of study designs, methodological quality criteria, and possible responses to each of the criteria. The second part provides definitions and explanations about the methodological quality criteria. After evaluating the 12 articles, all were determined to have met the MMAT criteria for inclusion and further analysis. Of the 12 articles included in this literature review, seven were qualitative and five were quantitative studies. These articles identified the factors that influence nurses' knowledge and attitudes toward patients in chronic pain with OUD. These studies were conducted in different countries: United States ( $\mathrm{n}=3)$, United Kingdom, $(\mathrm{n}=2)$, Jordan $(\mathrm{n}=1)$, India $(\mathrm{n}=1)$, Taiwan $(\mathrm{n}=1)$, Canada $(\mathrm{n}=1)$, Australia $(\mathrm{n}=1)$, and Norway $(\mathrm{n}=1)$.

\section{Findings}

Five main themes emerged from the 12 retrieved articles. These were (a) education, (b) role support, (c) nurses' experiences, (d) demographic factors, and (e) nurses' beliefs and culture. Twelve sub-themes were also identified. The five themes and subthemes are described below.

\subsection{Education}

Articles included in this literature review emphasized education as a key element that influences nurses' interactions with patients in chronic pain with OUD. It was highlighted in this review that education consists of nurses' level of education, school curriculum, post-graduation educational programs about pain management and OUD, and competency. A study by Chang and Yang (2013) ${ }^{[22]}$ reported education being a factor that potentially influenced nurses' attitudes toward patients with opioid abuse. They found that nurses with college or above degrees had more positive attitudes toward patients with opioid abuse compared to nurses with lower educational levels. The importance of nursing curriculum and post-graduation educational classes on the quality of nursing care to patients in chronic pain with opioid abuse has been studied extensively. For example, Monks, Topping, and Newell (2013) ${ }^{[23]}$ explored the abilities of 29 nurses to provide care to patients with drug abuse in North West Eng- land. These authors reported that none of study participants received pre- or post-registration education about managing patients with drug abuse. In consequence, these nurses lacked the knowledge and confidence in providing adequate care to these patients. Similar findings were reported in the study by Krokmyrdal and Andenaes (2015). ${ }^{[24]}$ They reported that nurses in medical and orthopedic units who had not attended any OUD and pain management classes affected their competency in managing chronic pain patients with OUD. For example, $80.3 \%$ of the study participants were unable to recognize pain in their patients. Similarly, a qualitative study by Siedlecki et al. (2014), ${ }^{[25]}$ involving 48 nurses working in medical-surgical units and emergency departments, explored their experience and education to manage chronic pain patients. These researchers reported that $77 \%(\mathrm{n}=37)$ nurses did not attend any pain management educational classes, and the majority had difficulty in distinguishing chronic from acute pain. Such inability resulted in inadequate care to patients with chronic pain. In other studies as well, nurses reported lacking the necessary knowledge and skills to provide care to patients in chronic pain with opioid addiction. ${ }^{[26,27]}$

However, Hamdan-Mansour, Mahmoud, Asqalan, Alhasana, and Alshibi (2012) ${ }^{[26]}$ found in their quantitative study no significant differences between emergency Jordanian nurses with higher academic levels and nurses with low academic levels regarding their knowledge to recognize patients with drug seeking behaviors. These authors also found no statistically significant difference in the knowledge between nurses who received or did not receive training in their practice about opioid use patients. Similarly, Chang and Yang $(2013)^{[22]}$ found that nursing school education had no significant difference in enhancing nurses' abilities to manage drug abuse patients.

\subsection{Role support}

Organizational and collegial support influences the nursing care that is provided to patients in chronic pain with OUD. Organizations may support or hinder the role of nurses who care for these patients. Morgan (2014) ${ }^{[27]}$ used grounded theory to understand the attitudes of 14 nurses toward patients with addiction in pain. This researcher found that organizational barriers to the nurses' role in caring for patients with drug abuse included shortage of staff, inability to contact specialized health care professionals, and lack of effective policies and mandatory pain education. Siedlecki et al. (2014) ${ }^{[25]}$ reported that staff shortage, system complexities, and organizational policies were the main reasons for ineffective pain management. They also found that nurses' workload affected their abilities to conduct proper pain assessment and provide 
effective pain interventions. Similar results were reported by Morley, Briggs, and Chumbley (2015). ${ }^{[28]}$ They identified that financial issues of organizations affected nursing resources that are needed for nursing education and professional development. In addition to resources, a health care organization is responsible to maintain safe work environments to protect and support health care providers in delivering high quality of care. In a qualitative study conducted in Australia by Ford (2011) ${ }^{[29]}$ showed that lack of safety and security measures for nurses caring for patients with drug addiction could make these nurses vulnerable, which in turn may impact their nursing care because they are under stress.

Chu and Galang (2013) ${ }^{[30]}$ showed that the presence of role support motivates nurses to work with patients who have addiction. They explained that nurses who receive advice and support from their colleagues had positive impacts on their clinical practice with these patients. However, this was not supported in a study conducted by Krokmyrdal and Andenaes (2015). ${ }^{[24]}$ These researchers found that collegial support did not contribute positively to nurses' competencies regarding pain management and opioid addiction.

\subsection{Demographic factors}

Nurses' age and their gender influence nurses' knowledge and attitudes to provide quality of care to patients in chronic pain with OUD. Studies have reported that there is a significant correlation between participants' age and their nursing practice with drug abuse patients and pain management. ${ }^{[22,26]}$ A quantitative study by Krokmyrdal and Andenaes (2015) ${ }^{[24]}$ examined the knowledge and competence of nurses, ranging in age between 22-54, in pain management in Norway. They reported that older nurses lacked knowledge related to pain management more than younger nurses. These differences were found because younger nurses had more internet skills to gain informal education and apply the latest evidencebased practice in their nursing care. On the other hand, Chu and Galang (2013) ${ }^{[30]}$ used a drug and drug problem perception questionnaire to examine the influence of nurses' age on their attitudes toward patients with addiction. These authors pointed out that nurses' age did not affect their attitudes toward these patients.

Gender differences in nurses determine their attitudes in delivering patients care. A study conducted by Prem et al. $(2011)^{[31]}$ used pain attitudes and beliefs scale to examine nurses' perception toward biomedical and behavioral dimensions of chronic pain. These authors found that female nurses had more positive attitudes toward patients' expression of pain compared to male nurses. On the other hand, Chu and Galang (2013) ${ }^{[30]}$ used a cross sectional design to examine gender as one of the factors that affects nurses' therapeutic attitudes toward patients with drug use. These authors found no significant differences in participants' behaviors toward these patients due to gender. Similarly, Hamdan-Mansour et al. $(2012)^{[26]}$ examined gender differences in nurses' knowledge who care for drug seeking patients in pain. They found that there was no difference between the gender of nurses and their knowledge of caring for drug seeking patients.

\subsection{Nurses' experiences}

Nurses' experiences about OUD and chronic pain may influence their knowledge and attitudes in managing chronic pain management. These experiences were shaped by years of nursing practice, areas of practice, and patients' behaviors related to pain management and opioid abuse. Some junior nurses struggled to manage these patients, while nurses with more years of experience showed better management. ${ }^{[22,26,28]}$ Morgan (2014) ${ }^{[27]}$ used grounded theory to interview hospital nurses who work with OUD patients in pain. This author found that nurses with more years of experience were able to effectively manage patients in pain with OUD. However, the results of Krokmyrdal and Andenae's $(2015)^{[24]}$ study indicated that the number of years of nursing practice had no significant effects on nurses' knowledge in pain management.

Nurses' areas of practice also have major impacts on the quality of nursing care. Chang and Yang (2012) ${ }^{[22]}$ reported that nurses who worked in mental health settings had more positive attitudes toward patients with opioid abuse than those in general hospital settings. These authors suggested that nurses in mental health departments were prepared and equipped with professional attitudes, knowledge, and skills that helped them to provide holistic care for these patients. In Krokmyrdal and Andenaes's (2015) ${ }^{[24]}$ study, workplace experience considered to be the most important influential factor on nurses' competencies in caring for OUD patients in chronic pain.

\subsection{Nurses' beliefs and culture}

Nurses' beliefs and culture are other factors that contribute to nursing management of patients with OUD in chronic pain. McCreaddie et al. (2010) $)^{[32]}$ and Morely et al. (2015) ${ }^{[28]}$ reported that stigmatization and stereotyping behaviors were present among some of the nurses in their study. These nurses described patients in pain with OUD as difficult, manipulative, aggressive, and generally challenging. These nurses' negative beliefs decreased the quality of nursing care and effective pain management. Nurses in a study by Neville and Roan $(2014)^{[33]}$ reported that they faced challenges in managing patients with drug abuse. Nurses reported that they experienced moral distress whether they should acknowl- 
edge the patients' self-report of pain or depend on their own observations for signs of pain. These challenges were also found in a study by Ford (2011) ${ }^{[29]}$ where nurses reported that it was difficult to believe patients' pain without any measurable evidence. Nurses also believed that their priority of care should be provided to patients who are admitted with critical physical illness rather than drug abuse. ${ }^{[23]}$ A qualitative study conducted by Siedlecki et al.(2014) ${ }^{[25]}$ in an acute care setting found participated nurses believed that patients with chronic pain abused pain scales assessment and asked for more narcotics; such behaviors led the nurses to view patients as drug seekers or abusers. Similarly, Ford (2011) ${ }^{[29]}$ reported that nurses perceived patients as drug seekers when they refused experts' pain medication prescriptions and insisted on demanding particular types of opioids. Krokmyrdal and Andenaes (2015) ${ }^{[24]}$ supported these findings in their study. The nurses in their study believed that patients with opioid addiction exaggerated their pain to get opioid medications. However, Morely et al. (2015) ${ }^{[28]}$ emphasized that each patient with drug abuse in pain is different and they need individualized holistic care.

In addition to nurses' beliefs, nursing care for patients with drug abuse may also be influenced by nurses' cultural backgrounds. In a qualitative study conducted by Monks et al (2013) ${ }^{[23]}$ in England participants reported that they labeled patients with drug abuse based on their social norms. The study participants labeled patients who had complications from drug use as addicts, which caused distrust in their assessment and management. Morgan (2014) ${ }^{[27]}$ conducted a qualitative study in the United States to explore nurses' attitudes and interactions with patients with opioids in pain. The study participants did not manage pain with opioids because in their culture opioids may cause addiction, and they did not want to encourage addiction in their community.

\section{Discussion}

This literature review aimed to highlight the factors that affect nurses' knowledge and attitudes toward patients' quality of care and effective pain management. Findings of this literature review will help nurses to understand factors that may influence their knowledge and attitudes toward patients in chronic pain with OUD. These findings will also provide some recommendations in enhancing nurses' knowledge and improving their attitudes toward these patients. Through thematic analysis, this literature review showed that nurses' knowledge and attitude are influenced by their education, role support, socio-demographic characteristics, work experience, and beliefs and culture.

Education is an important factor that defines nurses' knowledge and shapes their attitudes toward patients in chronic pain with OUD. Nurses' education influences their knowledge and attitudes and the quality of their care to patients in chronic pain with OUD. Our finding is consistent with other research that suggested a positive relationship between nurses' education and their willingness to improve their knowledge about pain management. ${ }^{[34]}$ Other research also suggested higher educational levels led to more positive attitudes toward any changes in clinical protocol about pain management. ${ }^{[35]}$ Continuing or in-service education by organizations to nurses about pain management helped them to effectively manage their patients' pain. ${ }^{[35]}$

However, while formal education provides nurses with knowledge that is needed to deliver care for patients in chronic pain with OUD, not all nursing education programs include adequate addiction studies in their curriculum which influenced nurses' ability to care for patients with addiction. ${ }^{[36]}$ A study demonstrated that a dedicated in-service education about pain management for nurses working in medical and surgical units led to a remarkable increase in their knowledge and attitudes with all types of pain management. ${ }^{[34]}$

However, nurses' knowledge alone is not sufficient to improve the quality of care they provide to patients in chronic pain with OUD. Nurses need to possess broader competencies in their nursing care. Competency is defined as the effective use of knowledge, skills, clinical reasoning, emotions, and reflection in the clinical setting for the benefit of the individuals and community being served. ${ }^{[37]}$ Our review highlighted the complexity of managing patients in chronic pain with OUD that nurses encounter in their daily practice. ${ }^{[24,26]}$ Therefore, nurses require special competencies to care for these patients effectively and promote their wellbeing. ${ }^{[38]}$ Competent nurses are also able to enhance the quality of care and protect their patients from inappropriate judgment and nursing practice. Our review underscored the essential necessity of nurses' competency to recognize chronic pain in OUD patients to ensure their effective pain management. The lack of competency in recognizing drugabusing patients in pain in emergency departments resulted in repeated visits of these patients seeking effective pain management. ${ }^{[39]}$ While our review showed a positive relationship between education and competence and quality patient care consistent with research studies, others showed contradicting results. ${ }^{[26,40]}$

Another factor that affects nurses' knowledge and positive attitudes toward patients in chronic pain with OUD that influenced the delivery of effective pain management was the role support that nurses received from their organizations and colleagues. This finding was supported in a study by Ford et 
al. $(2008)^{[6]}$ that showed the necessity of organizational and collegial support to build confidence in nurses in delivering nursing care. ${ }^{[6]}$ Lack of organizational and collegial support also led nurses to feel overwhelmed in providing high quality of care and effectively managing patients' pain. ${ }^{[42]}$ Improving nurses' attitudes through organizational support was also highlighted in our review that organizational support for health care professionals increases their self-esteem, satisfaction, and willingness to work with alcohol users to manage their pain. ${ }^{[11]}$

Our review found conflicting reports on the impact of age and gender on nurses' knowledge and attitudes toward patients in chronic pain with OUD. Studies by Manwere, Chipfuwa, Mukwamba, and Chironda (2015) and Pretorius, Searle, and Marshall (2015) reported positive associations with age. ${ }^{[43,44]}$ In contrast, other studies found no statistically significant correlation between age and nurses' knowledge and attitudes toward patients in chronic pain with OUD. ${ }^{[30,45]}$ With respect to gender, the majority of the studies included in our review reported no significant correlation between nurses' gender and their knowledge and attitudes toward patients in chronic pain with OUD. This was supported by the findings of Latina et al.'s (2015) ${ }^{[45]}$ study exploring male and female nurses' knowledge and attitudes toward quality pain management. Similar results were reported by Gilchrist et al. (2011). ${ }^{[46]}$

Our review as well showed conflicting results about the impact of nurses' experience on their knowledge and attitudes in the care of these patients. Several studies have shown positive associations between the frequency of exposure to patients with substance abuse and nurses' experience in pain management and improvements in patients' care and their experience of pain control. ${ }^{[40,45,47]}$ However, other studies in our review showed no significant relationship between nurses' years of experience with their knowledge and attitude toward opioid. This finding is in line with the findings in a study conducted by Costello and Thompson (2015) ${ }^{[48]}$ that assessed nurses' knowledge of opioid use and their years of experiences. These authors concluded that nurses' lack of sufficient training about pain management was a primary factor, not their years of nursing practice. Other studies as well have supported practice exposure being a contributing factor. For example, a study by Yava et al. (2013) ${ }^{[49]}$ found that nurses who worked in surgical units had more knowledge about pain management compared to others working in medical units because they frequently dealt with patients complaining of postoperative pain. Another example of practice exposure that results in negative attitudes in nurses is OUD patients' abusive behaviors when seeking care. ${ }^{[25,27]}$ A study by Natan, Beyil, and Neta (2009) $)^{[50]}$ reported on how patients' behaviors (e.g., not providing accurate medical histories or lying to get specific opioids only) formed their negative attitude toward them.

Finally, our review found that nurses' beliefs and culture as well play an important role in providing nursing care for patients in chronic pain with OUD. This is in alignment with findings in a study by Daibes et al. (2017). ${ }^{[36]}$ Negative attitudes toward these patients resulted in negative outcomes as reported by Gauntlett-Gilbert, Rodham, Jordan, and Brook (2015). ${ }^{[51]}$ These researchers found that nurses and physicians working in an emergency department believed that chronic pain was a chronic condition that did not require emergency interventions resulting in under-treatment of these patients' pain.

The cultural background of nurses also affects their knowledge and attitudes toward patients in chronic pain with OUD. A study in Jordan found that nurses stigmatized these patients because they considered them to be socially different and deviated from acceptable social and cultural standards. ${ }^{[36]}$ Religious misconceptions as well negatively influenced nurses' attitude towards these patients. ${ }^{[52]}$

In sum, thematic analysis of the peer-reviewed literature identified that nurses' knowledge and attitude toward patients in chronic pain with OUD are influenced by their education, role support, demographic characteristics, nurse's experiences and beliefs and culture.

\subsection{Strengths and limitations}

This literature review has several strengths. A systematic search using Cronin et al.'s (2008) ${ }^{[20]}$ method was used to find high quality of articles. Another strength of this literature review is that all included articles are current primary studies that were published between 2008 and 2019. Included articles examined the nurses' knowledge and attitudes in different populations with different contexts, such as United Kingdom, India, and Jordan. Some limitations were found also in this literature review. The articles included in this literature review were five quantitative studies and seven qualitative studies, but no mixed method studies. Another limitation was that quantitative articles included in this literature review used cross sectional or descriptive design, and convenient sampling, which may affect the generalizability of their findings. Another limitation is that only a few of the 12 articles entirely explored nurses' knowledge and attitude toward patients in chronic pain with OUD. Some studies were only about the nurses' knowledge and attitude toward patients in chronic pain; while others studied nurses' knowledge and attitude toward OUD alone. Another limitation is that all studies were conducted outside Qatar and no relevant 
articles were found in the Gulf region.

\subsection{Implications and recommendations}

This literature review pinpointed different aspects and influential factors of the issue of nurses' knowledge and attitude toward patients in chronic pain with OUD. Since nurses play a crucial role in identifying and managing chronic pain inOUD patients, their negative attitudes are unacceptable. It is important to modify the factors that affect nurses' attitudes toward care for this population, such as their lack of education about pain in OUD patients. Thus, it is recommended to develop and conduct an evidence-based educational program for nurses who frequently encounter these patients. This evidence-based educational workshop will improve nurses' attitudes, their knowledge, and skills in the recognition, assessment, care, and management of patients in chronic pain with OUD. The findings of this literature review emphasized the need for a study to investigate nurses' knowledge and attitudes as well the consequences of their attitude on the quality of patients' care would be of benefit in the context of Qatar.

\section{Conclusion}

In conclusion, nurses' knowledge and attitudes toward patients in chronic pain with OUD is a complex issue that impacts the quality of care and effectiveness of pain management. The aim of this literature review was to explore the factors that influence nurses' knowledge and attitudes toward this population. The main factors that have been reported in this literature review were nurses' education, role support, socio-demographic factors, nurses' experience, and nurses' beliefs and culture. The findings of this literature review will allow nurses to identify the gap in their clinical practice of recognizing and managing patients in chronic pain with OUD. Through the findings of this literature review, an educational workshop is proposed for nurses to improve their knowledge, attitudes, and skills in delivering of health care to these patients.

\section{CONFLicts OF InTEREST Disclosure}

The authors have no conflicts of interest.

\section{REFERENCES}

[1] Saxena A, Jain P, Bhatnaga $S$. The prevalence of chronic pain among adults in India. Indian. Journal of Palliative Care. 2018; 24(4): 472 477. PMid:30410260 https://doi.org/10.4103/IJPC.IJPC_ 141_18

[2] Zghoul N, Ross EL, Edwards RR, et al. Prevalence of chronic pain with neuropathic characteristics: A randomized telephone survey among medical center patients in Kuwait. Journal of Pain Research 2017; 17(S11). https ://doi.org/10.1016/j.jpain.2016.01 043

[3] Hagemeier N. Introduction to the opioid epidemic: The economic burden on the health care system and impact on quality of life. The American Journal of Managed Care. 2018; 24(10, suppl): S200-206

[4] World Health Organization. Information sheet on opioid overdose 2018. Available from: https://www. who.int/substanceabuse /information-sheet/en/

[5] Alam-mehrjerdi Z, Noori R, Dolan K. Opioid use, treatment and harm reduction services: The first report from the Persian Gulf region. Journal of Substance Use. 2016; 21(2): 217-223.

[6] Ford R, Bammer G, Becker N. The determinants of nurses' therapeutic attitude to patients who use illicit drugs and implications for workforce development. Journal of Clinical Nursing. 2008; 17(18): 2452-2462. PMid:18547349 https://doi.org/10.1111/j.13 65-2702.2007.02266.x

[7] Buchman DZ, Leece P, Orkin A. The epidemic as stigma: The bioethics of opioids. The Journal of Law, Medicine \& Ethics. 2017; 45(4): 607-620. https://doi.org/10.1177/10731105177506 00

[8] Pergolizzi JV, Gharibo C, Passik S, et al. Dynamic risk factors in the misuse of opioid analgesics. Journal of Psychosomatic Research. 2012; 72(6): 443. PMid:22656441 https://doi.org/10.1016/ j.jpsychores.2012.02.009
[9] Mascola MA, Borders AE, Terplan M. Committee opinion No. 711: Opioid use and opioid use disorder in pregnancy. Obstetrics \& Gynecology. 2017; 130(2): 488-489. PMid:28742670 https : //doi.org/10.1097/A0G.0000000000002229

[10] Cheatle M, Comer D, Wunsch M, et al. Treating pain in addicted patients: Recommendations from an expert panel. Population Health Management. 2014; 17(2): 79-89. PMid:24138341 https://doi.org/10.1089/pop. 2013.0041

[11] Voon P, Karamouzian M, Kerr T. Chronic pain and opioid misuse: A review of reviews. Substance Abuse Treatment, Prevention \& Policy. 2017; 12(1-9). PMid:28810899 https://doi.org/10.1186/s1 3011-017-0120-7

[12] Gregg JA, Jones JS. How do you recognize opiate addiction in the rehabilitation patient?. Rehabilitation Nursing. 2008; 38(5): 217-220. PMid:23696468 https : //doi.org/10.1002/rnj.90

[13] Ashley JL. Pain management: Nurses in jeopardy. Oncology Nursing Forum. 2008; 35(5): E70-5. https ://doi.org/10.1188/08. ONF .E70-E75

[14] Kahan M, Wilson L, Wenghofer EF, et al. Pharmacists' experiences with dispensing opioids: Provincial survey. Canadian Family Physician. 2011; 57(11): e448-54.

[15] Peppin JF, Passik SD, Couto JE, et al. Recommendations for urine drug monitoring as a component of opioid therapy in the treatment of chronic pain. Pain Medicine. 2012; 13(7): 886-896. PMid:22694154 https://doi.org/10.1111/j.1526-4637.2012.01414.x

[16] Gong CZ, Liu W. Acupuncture and the opioid epidemic in American Chinese. Journal of Integrative Medicine. 2018; 24(5): 323-327. PMid:29752610 https : //doi .org/10.1007/s11655-018-298 $9-z$

[17] Griffiths D. The role of the nurse in preventing opioid abuse. Ohio Nurses Review. 2017; 92(3): 14-15. 
[18] Hudspeth RS. Safe opioid prescribing for adults by nurse practitioners: Part 1.Patient history and assessment standards and techniques. The Journal for Nurse Practitioners. 2016; 12(3): 141-148. https://doi.10.1016/j.nurpra.2015.11.032

[19] Hasselt M, Keyes V, Bray J, et al. Prescription drug abuse and workplace absenteeism: Evidence from the 2008-2012 national survey on drug use and health. Journal of Workplace Behavioral Health. 2015; 30(4): 379-392. https://doi .org/10.1080/15555240.2 015.1047499

[20] Cronin P, Ryan F, Coughlan M. Undertaking a literature review: A step-by-step approach. British Journal of Nursing. 2008; 17(1): 3843. PMid:18399395 https://doi.org/10.12968/bjon. 2008.1 7.1 .28059

[21] Hong QN, Pluye P, Fabregues S, et al. Mixed Methods Appraisal Tool (MMAT), version 2018. Registration of Copyright (\#1148552), Canadian Intellectual Property Office, Industry Canada.

[22] Chang YP, Yang MS. Nurses' attitudes toward clients with substance use problems. Perspectives in Psychiatric Care. 2013; 49(2): 94-102. PMid:23557452 https://doi.org/10.1111/ppc.12000

[23] Monks R, Topping A, Newell R. The dissonant care management of illicit drug users in medical wards, the views of nurses and patients: A grounded theory study. Journal of Advanced Nursing. 2013; 69(4): 935-946. PMid:22776007 https://doi .org/10.1111/j. 1365-2648.2012.06088.x

[24] Krokmyrdal KA, Andenæs R. Nurses' competence in pain management in patients with opioid addiction: A cross-sectional survey study. Nurse Education Today. 2015; 35(6): 789-794. PMid:25779029 https://doi.org/10.1016/j.nedt.2015.02.022

[25] Siedlecki SL, Modic MB, Bernhofer E, et al. Exploring how bedside nurses care for patients with chronic pain: A grounded theory study. Pain Management Nursing. 2014; 15(3): 565-573. PMid:23466194 https://doi.org/10.1016/j.pmn.2012.12.007

[26] Hamdan-Mansour AM, Mahmoud KF, Asqalan SM, et al. Knowledge and practice of Jordanian emergency nurses caring for patients with drug-seeking behaviors. Clinical Nursing Research. 2012; 21(3): 368384. PMid:21878582 https://doi.org/10.1177/1054773811 420284

[27] Morgan BD. Nursing attitudes toward patients with substance use disorders in pain. Pain Management Nursing. 2014; 15(1): 165175. PMid:24602434 https://doi.org/10.1016/j.pmn. 2012 .08 .004

[28] Morley G, Briggs E, Chumbley G. Nurses' experiences of patients with substance-use disorder in pain: A phenomenological study. Pain Management Nursing. 2015; 16(5): 701-711. PMid:25979457 https://doi.org/10.1016/j.pmn.2015.03.005

[29] Ford R. Interpersonal challenges as a constraint on care: The experience of nurses "care of patients who use illicit drugs.Contemporary Nurse. 2011; 37(2): 241-252. PMid:21692595 https://doi.org/ $10.5172 /$ conu. 2011.37 .2 .241

[30] Chu C, Galang A. Hospital nurses' attitudes toward patients with a history of illicit drug use. Canadian Nurse. 2013; 109(6): 29-34.

[31] Prem V, Karvannan H, Chakravarthy RD, et al. Attitudes and beliefs about chronic pain among nurses- biomedical or behavioral? A cross-sectional survey. Indian Journal of Palliative Care. 2011; 17(3): 227-234. PMid:22346048 https://doi .org/10.4103/09 73-1075. 92341

[32] McCreaddie M, Lyons I, Watt D, et al. Routines and rituals: A grounded theory of the pain management of drug users in acute care settings. Journal of Clinical Nursing. 2010; 19(19/20): 2730-2740. PMid:20846223 https://doi.org/10.1111/j.1365-2702.20 $10.03284 . \mathrm{x}$

Published by Sciedu Press
[33] Neville K, Roan N. Challenges in nursing practice: Nurses' perceptions in caring for hospitalized medical-surgical patients with substance abuse/dependence. Journal of Nursing Administration. 2014; 44(6): 339-346. PMid:24835142 https://doi .org/10.1097/NN A. 0000000000000079

[34] Keen A, McCrate B, McLennon S, et al. Influencing nursing knowledge and attitudes to positively affect care of patients with persistent pain in the hospital setting. Pain Management Nursing. 2017; 18(3): 137-143. PMid:28528935 https://doi.org/10.1016/j . pmn.2017.04.002

[35] Hadorn F, Comte P, Foucault E, et al. Task-shifting using a pain management protocol in an emergency care service: Nurses' perception through the eye of the Rogers's diffusion of innovation theory. Pain Management Nursing. 2016; 17(1): 80-87. PMid:26602151 https://doi.org/10.1016/j.pmn.2015.08.002

[36] Daibes MA, Al-Btoush MS, Marji T, et al. Factors influencing nurses' attitudes towards patients in Jordanian addiction rehabilitation centers: A qualitative study. International Journal of Mental Health and Addiction. 2017; 15(3): 588-603. https://doi.org/10.1007/s1 1469-016-9682-2

[37] Valizadeh L, Zamanzadeh V, Eskandari M, et al. Professional competence in nursing: A hybrid concept analysis. Medical-Surgical Nursing Journal. 2019; 8(2): 1-8. https://doi .org/10.5812/ms $\mathrm{nj} .90580$

[38] Lovi R, Barr J. Stigma reported by nurses related to those experiencing drug and alcohol dependency: A phenomenological Giorgi study. Contemporary Nurse. 2009; 33(2): 166-178. PMid:19929161 https://doi.org/10.5172/conu.2009.33.2.166

[39] Bystrek DJ. Emergency department doctors' and nurses' knowledge and attitudes concerning substance use and users. Journal of Trauma Nursing. 2010; 17(2): 119-120. https ://doi.org/10.1097/JTN . Ob013e3181e737ce

[40] Eid T, Manias E, Bucknall T, et al. Nurses' knowledge and attitudes regarding pain in Saudi Arabia. Pain Management Nursing. 2014; 15(4): e25-36. PMid:25179424 https://doi.org/10.101 6/j.pmn.2014.05.014

[41] Van Boekel LC, Brouwers PM, Van Weeghel J, et al. Stigma among health professionals towards patients with substance use disorders and its consequences for health care delivery: Systematic review. Drug \& Alcohol Dependence. 2013; 131(1/2): 23-35. PMid:23490450 https://doi.org/10.1016/j.drugalcdep.2013.02.018

[42] Bergman CL. Emergency nurses' perceived barriers to demonstrating caring when managing adult patients' pain. Journal of Emergency Nursing. 2012; 38(3): 218-225. PMid:22578392 https: //doi.org/10.1016/j.jen.2010.09.017

[43] Manwere A, Chipfuwa T, Mukwamba MM, et al. Knowledge and attitudes of registered nurses towards pain management of adult medical patients: A case of Bindura hospital. Health Science Journal. 2015; 9(4).

[44] Pretorius A, Searle J, Marshall B. Barriers and enablers to emergency department nurses' management of patients' pain. Pain Management Nursing. 2015; 16(3): 372-379. PMid:25440235 https: //doi.org/10.1016/j.pmn.2014.08.015

[45] Latina R, Mauro L, Mitello L, et al. Attitude and knowledge of pain management among Italian nurses in hospital settings. Pain Management Nursing. 2015; 16(6): 959-967. PMid:26697820 https: //doi.org/10.1016/j.pmn.2015.10.002

[46] Gilchrist G, Moskalewicz J, Slezakova S, et al. Staff regard towards working with substance users: AEuropean multi-centre study. Addiction. 2011; 106(6): 1114-1125. http://doi-org.ezproxy.1 ib.ucalgary.ca/10.1111/j.1360-0443.2011.03407.x 
[47] Al-Shaer D, Hill PD, Anderson MA. Nurses' knowledge and attitudes regarding pain assessment and intervention. Medsurg Nursing. 2011; 20(1): 7-11.

[48] Costello M, Thompson S. Preventing opioid misuse and potential abuse: The nurse's role in patient education. Pain Management Nursing. 2015; 16(4): 515-519. PMid:25497402 https://doi.org/10 $.1016 / j$. pmn. 2014.09.008

[49] Yava A, Çicek H, Tosun N, et al. Knowledge and Attitudes of Nurses about Pain Management in Turkey. International Journal of Caring Sciences. 2013; 6(3): 494-505.

[50] Natan MB, Beyil V, Neta O. Nurses' perception of the quality of care they provide to hospitalized drug addicts: Testing the theory of reasoned action. International Journal of Nursing Practice (Wiley-Blackwell). 2009;15(6): 566-573. PMid:19958412 https: //doi.org/10.1111/j.1440-172X.2009.01799.x

[51] Gauntlett-Gilbert J, Rodham K, Jordan A, et al. Emergency department staff attitudes toward people presenting in chronic pain: A qualitative study. Pain Medicine. 2015; 16(11): 2065-2074. PMid:26177229 https://doi.org/10.1111/pme.12844

[52] Al Qadire M, Al Khalaileh M. Jordanian nurses knowledge and attitude regarding pain management. Pain Management Nursing. 2014; 15(1): 220-228. PMid:24602438 https://doi.org/10.1016/j . pmn.2012.08.006 